\title{
Frontières
}

\section{Anxiété de mort, psychosexualité et transition de retraite}

\section{Jocelyne Thériault}

Volume 14, numéro 2, printemps 2002

La mort prononcée

URI : https://id.erudit.org/iderudit/1073976ar

DOI : https://doi.org/10.7202/1073976ar

Aller au sommaire du numéro

Éditeur(s)

Université du Québec à Montréal

ISSN

1180-3479 (imprimé)

1916-0976 (numérique)

Découvrir la revue

Citer cet article

Thériault, J. (2002). Anxiété de mort, psychosexualité et transition de retraite. Frontières, 14(2), 73-77. https://doi.org/10.7202/1073976ar

\section{Résumé de l'article}

Cet article porte sur le phénomène de l'anxiété de mort lors du passage travail-retraite. Il propose d'abord de définir la notion d'anxiété de mort, puis d'explorer le phénomène d'anxiété de mort à partir de l'histoire préretraite, intraretraite et postretraite de trois travailleurs manuels engagés dans le processus de transition travail-retraite. Ces trois histoires personnelles sont issues d'un échantillon d'histoires personnelles provenant d'une étude longitudinale portant sur la transition à la retraite. D'un point de vue pratique, cette étude contribue à cerner les besoins des gens en cours de transition à la retraite et contribue, ce faisant, à identifier les pistes à privilégier pour mieux intervenir auprès d'eux.
Ce document est protégé par la loi sur le droit d'auteur. L'utilisation des services d’Érudit (y compris la reproduction) est assujettie à sa politique d'utilisation que vous pouvez consulter en ligne.

https://apropos.erudit.org/fr/usagers/politique-dutilisation/ 


\section{Résumé}

Cet article porte sur le phénomène de l'anxiété de mort lors du passage travailretraite. II propose d'abord de définir la notion d'anxiété de mort, puis d'explorer le phénomène d'anxiété de mort à partir de I'histoire préretraite, intraretraite et postretraite de trois travailleurs manuels engagés dans le processus de transition travail-retraite. Ces trois histoires personnelles sont issues d'un échantillon d'histoires personnelles provenant d'une étude longitudinale portant sur la transition à la retraite. D'un point de vue pratique, cette étude contribue à cerner les besoins des gens en cours de transition à la retraite et contribue, ce faisant, à identifier les pistes à privilégier pour mieux intervenir auprès d'eux.

Mots clés : anxiété de mort - transition travail-retraite

\section{Abstract}

This article concerns the phenomenon of death anxiety during the workretirement transition period. It is first a proposal to define the notion of death anxiety and to demonstrate theoretic and empirical evidence from documentation on the subject. This article is secondly a proposal for exploring the phenomenon of death anxiety by using pre-retirement, intra-retirement, and post-retirement histories of three older employees involved in the work-retirement transition process. These three personal stories stem from a sampling of personal stories from a longitudinal study on the transition to retirement. From a practical point of view, this study contributes to identifying the needs of people in the retirement transition and in doing so also contributes to pinpointing leads to favor so as to intervene better on their behalf.

Key words : anxiety of death - transition work-retirement

\section{ANXIÉTÉ DE MORT,
PSYCHOSEXUALITÉ ET TRANSITION DE RETRAITE}

Jocelyne Thériault, Ph.D.,

professeure, Département de sexologie, UQÀM.

Cet article a pour but de mettre en lumière un phénomène qui reste souvent dans l'ombre dans la documentation portant sur la transition à la retraite et qui, pourtant, semble se profiler dans l'expérience des personnes traversant le passage travail-retraite. Il s'agit de l'anxiété de mort. Questionnés sur le sens du travail et le sens de la retraite dans leur vie, les pré-retraités et retraités que j'ai rencontrés ont utilisé l'occasion des rencontres de recherche pré-, intra- et postretraite pour introduire, dans leurs récits d'entrevue, leurs pensées et préoccupations au sujet de la mort. C'est en analysant longitudinalement leurs pensées et préoccupations de mort en relation avec leurs niveaux pré-, intra- et postretraite d'anxiété générale (recueillis au test IPAT: Cormier, 1962) que l'hypothèse d'une anxiété de mort accompagnant la transition travail-retraite fut posée.

Pour mettre en lumière ce phénomène, cet article propose d'abord de définir, en soi, la notion d'anxiété de mort et de présenter les faits théoriques et empiriques de la documentation sur le sujet. Ensuite, cet article propose d'explorer le phénomène d'anxiété de mort à partir de l'histoire préretraite, intraretraite et postretraite de trois travailleurs âgés engagés dans le processus de transition travail-retraite. Le design expérimental de l'étude longitudinale qui sert d'assise à cet article et l'échantillon total de sujets duquel proviennent ces trois histoires personnelles sont, à cet effet, brièvement présentés. Enfin, cet article débouche sur quelques considérations portant sur l'adaptation psychosexuelle et sociale des préretraités et retraités en situa- tion de passage travail-retraite. Ces considérations sont formulées en lien avec leurs pensées et préoccupations de mort.

Cette étude explorant l'anxiété de mort lors de la transition travail-retraite semble importante pour plusieurs raisons. D'une part, parce que l'expérience intrapsychique inhérente à la transition travail-retraite est peu connue (Guillemard, 1980; Elder, 1995). D'autre part, parce que les études sur la transition travail-retraite sont l'enfant pauvre de la documentation sur l'anxiété de mort, laquelle cible les personnes âgées sans distinction aucune des phases de retraite dans lesquelles elles sont inscrites. Enfin, il est important de clarifier le lien entre anxiété de mort et retraite afin de mieux intervenir (psychosexuellement, psychosocialement) auprès des personnes préretraités et nouvellement retraitées.

\section{ANXIÉTÉ DE MORT : DÉFINITION}

Quand vient le temps de définir l'anxiété de mort, plusieurs problèmes surgissent. En dépit de la quantité d'articles qui ont été publiés depuis la fin des années 1970 sur l'anxiété de mort, période où l'intérêt pour la question était à son zénith (Wass et al., 1987), la définition de l'anxiété de mort reste vague et diffuse. Dans la documentation scientifique, les expressions anxiété de mort et peur de la mort sont tantôt définies isolément, tantôt utilisées indistinctement. Kalish (1987) dira que là où la peur doit avoir un objet concret, l'anxiété n'en a pas. La peur serait une réaction à un danger externe spécifique alors que l'expérience de l'anxiété serait plus liée à des conflits internes, inconscients. Wass et al. (1987) concluent que la distinction entre les termes anxiété et peur de mort est futile puisqu'elle ne repose sur aucune base théorique ou 
empirique. Dans ce contexte, toute réaction émotionnelle négative face à la mort sera définie par l'un ou l'autre des deux termes. Ces réactions sont tantôt conscientes, tantôt inconscientes. Par exemple, les adultes plus âgées (60 ans et plus) montreraient des degrés plus élevés de peur ouverte de la mort que les jeunes adultes qui couvrent davantage leurs peurs de la mort (Galt et Hayslip, 1998). Les premiers comparés aux seconds ressentiraient moins le besoin de nier la peur de leur mort et celle des autres significatifs (Galt et Hayslip, 1998). Ces données contredisent la majorité des recherches passées montrant que l'anxiété de mort tend à décroître avec l'âge (Wass et al., 1987). Becker (1973) nuance ces dires en soulignant que les hommes vieillissants seraient plus portés que les femmes à nier la mort. De grands théoriciens existentialistes disent que l'anxiété de mort devient plus claire à mesure que l'individu avance en âge (Rappoport et al., 1993). La mort donnerait un sens à la vie plutôt que de la vider de tout son sens. S'en défendre - en ne mettant l'accent que sur la jeunesse, en prétendant qu'il n'y a pas de fin à la vie obscurcirait, chez l'individu, la perception de sa non-immortalité et l'empêcherait de rendre signifiant son temps de vie (Rappoport et al., 1993). D'autre part, des niveaux très élevés d'anxiété de mort seraient associés à une perception fermée $\mathrm{du}$ futur. Le futur devient inaccessible et l'espoir nécessaire pour construire le présent disparaît (Rappoport et al., 1993). Le désir déserte les lieux.

$\mathrm{Au}$ terme de ce survol, il importe de mettre en relief certaines limites rencontrées dans la documentation sur l'anxiété de mort. D'une part, elle a suscité peu ou pas d'études sur la personne en situation de transition travail-retraite ${ }^{1}$. Certes les écrits ont ciblé différentes populations de personnes âgées, mais elles n'ont pas ciblé les personnes en phase d'entrée dans la période de retraite. D'autre part, les recherches dans le domaine ont été de type transversal et ont été menées au moyen de questionnaires de recherche. Wass et al. (1987) recommandent les recherches longitudinales (menées à travers l'espace de vie des individus) plutôt que transversales dans l'investigation de l'anxiété de mort. Ils recommandent également l'entrevue et non seulement le questionnaire de recherche comme outil de mesure. Le présent article fait quelques pas en ce sens. Il rapporte trois histoires de transition de retraite qui sont tirées des données d'une étude longitudinale (Thériault, 1986) réalisée par entrevues et tests psychométriques auprès d'individus rencontrés à différents points de leur espace de vie - en préretraite et à l'entrée de la retraite.
Avant de présenter les histoires de retraite, il importe de décrire brièvement l'étude longitudinale à la base de ces récits. Le lecteur trouvera dans les travaux de Thériault (1994, 1986) la description détaillée du design expérimental et la méthodologie de cette étude. Rapportons ici simplement ces faits : l'étude longitudinale fut conduite auprès de travailleurs manuels en situation de retraite. Une double approche évaluative - quantitative et qualitative - fut utilisée. Cette recherche longitudinale voulait retracer certaines des composantes psychosociales significativement associées au processus de transition

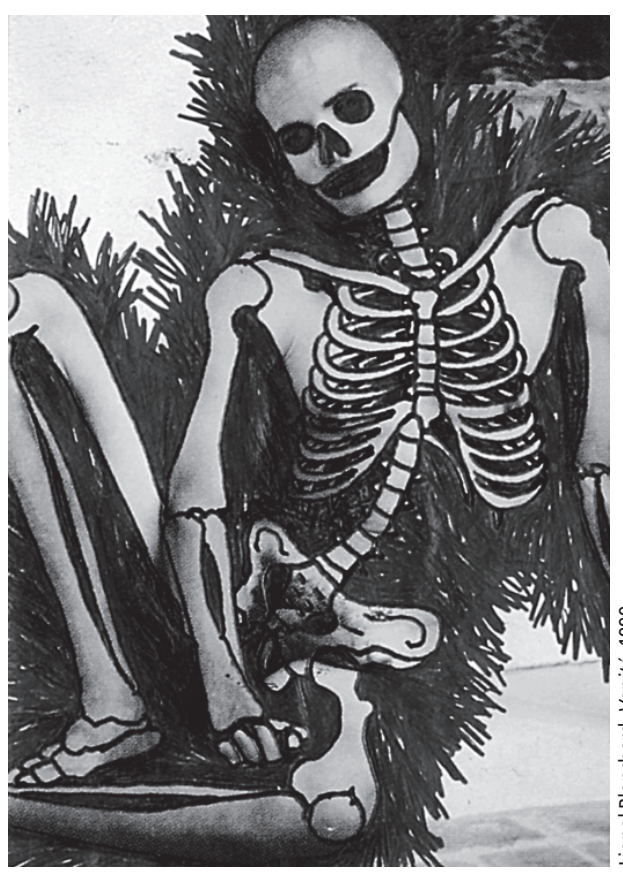

travail-retraite. Au total 39 sujets, âgés de 65 ans au moment du départ du travail, participèrent à l'étude (17 sujets du groupe expérimental rencontrés en pré-, intra- et postretraite $^{2}$ et 22 sujets du groupe contrôle rencontrés qu'en postretraite). Deux tests psychométriques connus (dont le test d'anxiété générale IPAT : Cormier, 1962) et l'entrevue clinique enregistrée furent utilisés à chacun des temps de mesure pré-, intraet postretraite. Au plan quantitatif, les résultats d'analyse de variance intermoments (ANOVA) montrent une variation significative pré-, postretraite des scores d'anxiété. Au plan qualitatif, les résultats d'analyse de contenu des entrevues cliniques enregistrées font ressortir l'existence de variations significatives pré-, postretraite dans les préoccupations rattachées à la mort. C'est l'analyse comparative de ces deux ensembles de données recueillies auprès de l'ensemble des sujets du groupe expérimental qui a permis de conclure en l'existence de l'anxiété de mort.

\section{TROIS HISTOIRES DE TRANSITION TRAVAIL-RETRAITE}

L'analyse de contenu de l'ensemble des entrevues de recherche longitudinale a permis de relever, parmi les récits étudiés, trois histoires personnelles de transition de retraite. Ces histoires personnelles furent choisies parce qu'elles sont jugées représentatives des différentes réactions des sujets rencontrés lors de cette investigation du passage travail-retraite. Chacune retrace à sa façon l'évolution des pensées et affects de mort lors du passage travail-retraite.

\section{MONSIEUR A.}

Un an avant la date prévue de son départ, Monsieur A. est atteint d'étourdissements importants justifiant la prescription par le médecin d'un arrêt complet du travail. Monsieur A. refuse l'arrêt de travail. Il précisera lors d'une dernière rencontre d'évaluation qu'à son avis cette maladie est imputable au fait qu'il a reçu de son employeur l'avis officiel de sa mise à la retraite : «ça m'a rendu malade», dira-t-il.

Lors de la première rencontre de recherche (six mois avant la date prévue de la retraite), Monsieur A. affiche un air assuré quoique ses propos soient très anxieux. L'avènement de cette maladie l'amène à remettre en question la retraite qu'il avait planifiée voilà dix ans déjà. Son univers anticipatif est maintenant rempli de doutes. Chacun de ses anciens projets de retraite s'alourdit du poids de craintes nouvelles. L'incertitude face au futur est au premier plan de son discours. Monsieur A. ne veut plus la retraite, il ne veut pas laisser derrière lui le travail. Il réalise maintenant que «le travail est normal à l'homme». Il prend conscience de toute sa dimension structurante. Le travail, dans ce contexte préretraite, est intrinsèque à la satisfaction de vie, voire à la survie de Monsieur A. Pour lui, l'arrêt du travail représente une chose : le spectre de la mort: "J'vas-tu me laisser mourir ? » C'est l'arrêt du travail entraîné par la retraite obligatoire et non la maladie qui engendre, à ses dires, ses anxiétés et menaces associées à la mort. Chez Monsieur A., la perspective de la retraite est donc porteuse de grandes craintes verbalisées. Le futur semble fermé ou du moins, il rend perplexe. La peur de la mort est au premier plan.

$\mathrm{Au}$ deuxième moment de rencontre, c'est-à-dire quelques semaines après la prise de la retraite, Monsieur A. formule moins de doutes. Beaucoup de divertissements ont estompé la réalité de sa retraite. Il se sent soulagé, lui semble-t-il. Par ailleurs il dira qu'il sent un manque, tout en ne sachant pas comment le définir. Il se demande si c'est le manque du travail comme tel ou le manque des rituels qui lui sont associés 
(sortir et revenir quotidiennement au foyer). Il ressent de façon évidente le changement de rythme occasionné par l'arrêt de travail : «C'est comme le jour et la nuit », dira-t-il. Il compte prendre certaines mesures pour s'adapter le mieux possible à la retraite. Il parle ici d'un régime alimentaire à suivre pour ne pas gagner trop de poids et ainsi risquer le pire. Monsieur A. compte sur son projet de voyage, sur la présence de son épouse, sur ses amis (élément nouveau dans son discours), pour l'aider à occuper son esprit et à se distraire de façon satisfaisante durant la retraite. Fait à noter, Monsieur A., tout comme la majorité des sujets rencontrés lors de cette deuxième entrevue, se dit en vacances plutôt que dans la période de retraite. Il pense encore au travail, mais il ne veut pas retourner voir ses anciens collègues. Il ne se comprend pas sur ce point; il trouve curieux cette attitude de sa part, lui qui a toujours entretenu de bons échanges avec ces personnes. Sa préoccupation majeure à cette étape est d'occuper son temps tout en étant entouré.

En résumé, à cette deuxième rencontre, Monsieur A. est moins soucieux de son devenir. Il s'embête lorsqu'il ne trouve rien à faire, mais il se rassure en se disant qu'il est en vacances (situation connue), et après, on verra. L'accent est mis sur le besoin de se distraire et il en est fort conscient. De quoi cherche-t-il à se distraire ? Des émotions et pensées associées aux pertes du passé (travail, collègues de travail, etc.) et à celles du futur, voire la mort? Quoi qu'il en soi, il compte sur les gens significatifs de son environnement et sur les projets à venir pour satisfaire ce besoin de distraction.

La troisième rencontre, sept mois après son départ du travail, est placée sous le signe de la délivrance, de la fierté. Monsieur A. se dit satisfait de sa retraite. Il ne voit pas le temps passer tant il est occupé. Il ne repense plus au travail passé. Comme s'il n'avait jamais travaillé, rétorque-t-il. Son épouse est régulièrement présente auprès de lui. Il songe occasionnellement à se trouver un emploi afin de se sentir entouré. Toutefois, ce projet semble moins impératif qu'avant. Il a également révisé ses grands projets de construction domiciliaire: il se trouve trop âgé pour entreprendre pareille aventure, cela dit sans amertume.

L'avenir lui semble riche de projets à poursuivre. Il est dorénavant orienté vers l'avant. Autant l'idée de mort le hantait six mois avant la retraite, autant il semble rassuré lors de cette dernière rencontre. «Serait-ce de vous en avoir parlé, me dit-il, serait-ce le fait d'avoir discuté de cela avec mon médecin, mais ce n'est plus pareil. »Monsieur A. a fait ses preuves et se montre fier de lui. Son rythme de vie semble lui convenir, et ce, pour aussi long- temps qu'il en aura la santé, précise-t-il. Par ailleurs, il dit ne pas penser à la mort et surtout ne pas vouloir y penser. Il veut vivre éternellement même si de façon réaliste " on sait tous que cela va arriver», se contente-t-il d'ajouter.

\section{MONSIEUR B.}

Lors de notre première rencontre de recherche, Monsieur B. perçoit la retraite qui s'annonce avec sérénité. Il n'anticipe aucun bouleversement important. Quoique le travail représente à ses yeux une « valeur de vie première», «une heureuse obligation », «une occupation de l'esprit» fort importante, la retraite signifie à la fois une obligation, une réalité inévitable mais aussi un moment désiré. C'est une étape de repos, mais surtout de grande liberté. Par le passé, cette éventualité de retraite le menaçait ditil : qu'allait-il faire? Depuis quelques mois, l'horaire de travail lui pèse de plus en plus et, depuis peu, la retraite ne le menace plus. Il a hâte à sa retraite: "J'aime autant la prendre ». Chez Monsieur B., peu de projets sont esquissés à cette période. Il veut éviter, ce faisant, les désillusions. Il est formel sur ce point. Trop souvent les décès, maladies ou autres limitations majeures empêchent les nouveaux retraités de réaliser leurs projets de retraite. Alors autant ne pas faire de projets. Il compte bien, par ailleurs, rester actif. À ses dires et aux dires d'une majorité de sujets rencontrés, un grand nombre de travailleurs manuels sont décédés au lendemain de leur départ du travail, et ce parce qu'ils étaient physiquement inactifs. Il veut donc occuper son temps et rester vigilant. À ce point-ci, il ne peut, par ailleurs, préciser de quelle façon il occupera ce temps, hormis les travaux domestiques, la lecture et les courses. L'avenir est perçu en termes d'une autre vie. Monsieur B. ne s'attarde toutefois pas à en tracer les contours. «On peut pas le savoir», reprend-il. Un désir perce néanmoins: il ne veut donner de «troubles» à personne.

Lors de la deuxième rencontre, Monsieur B. se sent perdu par grands moments. Il s'ennuie et ne sait que faire. Il est inquiet et ne sait pas comment réagir à cet état. $\mathrm{La}$ liberté tant souhaitée avant la retraite est remise en cause une fois la retraite arrivée. "On a beaucoup de temps libre, hein, pis, on n'est pas habitué. » Il se cherche. C'est à cette étape qu'il prend conscience de toute la valeur structurante de son travail. Il en parle en termes d'habitude: l'habitude de partir à telle heure, de préparer le « lunch» à telle heure, d'arriver au travail à telle heure pour revenir à la maison à telle heure passée. Tant d'occupations qu'il faut remplacer par autre chose maintenant, réaliset-il. Il ne désespère pas pour autant. Il mise sur le temps. L'adaptation s'en suivra, souhaite-t-il. Quoi qu'il en soit, Monsieur B. répète qu'il est content de ne plus travailler. «C'est fini, c'est fini » reprend-il avec insistance. À cette étape de la transition, Monsieur B. perçoit sa retraite non plus seulement en termes de repos, de vacances. Une nouvelle dimension s'ajoute : l'arrivée de la retraite signale sa finitude; la mort sera éventuellement au rendez-vous. [...] «Le dernier casier là, c'est l'dernier là, ça fait quelque chose. » Ce deuxième temps de la rencontre est caractérisé par l'ambivalence et l'indécision.

À la troisième rencontre Monsieur B. affirme catégoriquement qu'il n'aime pas son vécu de retraite. Ses perceptions préretraite se sont modifiées. Catégoriquement, il affirme que sa retraite est comme il l'avait imaginée, à savoir embêtante. Parallèlement, l'importance du travail revient au premier plan. Jadis, il anticipait avec hâte sa retraite. Maintenant, il critique les autorités privant les gens de travailler après 65 ans. La liberté devrait être laissée aux travailleurs âgés (rester s'ils le veulent à leur emploi après 65 ans) dit-il. Lors de cette troisième rencontre, Monsieur $\mathrm{B}$. semble toujours osciller entre deux états : une insatisfaction clairement verbalisée de son vécu actuel et une motivation peu affirmée à changer ce vécu.

Chez Monsieur B., il semble que l'évolution de ses pensées de mort - entre les phases pré- et postretraite - revêt une forme différente de celle retrouvée chez Monsieur A. Il semble y avoir décalage. Bien que les pensées de mort soient chez lui fort présentes au temps pré-retraite, elles ne semblent pas avoir mobilisé ses affects, comme ce fut le cas pour Monsieur A. Au temps intra-retraite, toutefois, Monsieur B. semble mobilisé par des affects dérangeants : ennui, inquiétudes, sentiment d'égarement. Il parle de sa propre finitude, la mort au bout de l'étape en cours. Au temps postretraite, le sens du travail et de la retraite est profondément réévalué; les émotions troubles semblent plus vives.

\section{MONSIEUR C.}

Lors de notre première rencontre de recherche, Monsieur C. critique son syndicat et son employeur. Il aime toutefois son travail. Il ne voit pas les journées passer au travail tant sa fonction requiert minutie et attention. Il jouit de son expérience de travail, voire des compétences qu'il a gagnées au fil du temps. Ces acquis le valorisent. «Le métier est là depuis tellement de temps, ça fait partie de notre vie, je crois. »Interrogé sur ses impressions concernant la retraite qui s'annonce, Monsieur C. dit ne pas y penser, voire n'en penser rien du tout. Il vit la journée comme elle vient. Il insiste pour dire que le spectre de la mortalité pré- et 
postretraite suffit à lui rappeler que tout doit se vivre au mieux la journée même. Sans être crainte, sans susciter de préoccupations apparentes, la mort se profile à l'horizon de son discours sur la retraite. Il présente une acceptation quasi inconditionnelle de la retraite à venir et de la mort éventuelle. «Tout ce qui naît passe, alors pourquoi s'en faire? On va tous passer par là ... on vient, on passe. Si jamais la maladie prend, ben coudon, ce sera comme les autres. »

Rencontré, dans un deuxième temps, quelques semaines après son départ officiel à la retraite, Monsieur C. prend tout à coup conscience de toute l'importance qu'avait son travail dans sa vie. "En arrêtant, on perd les copains, les discussions, les contacts chargés, pus de contacts de ce côté-là.» N'ayant plus ce travail, tout le mode de vie change, dit-il, «un nouvel équilibre est à refaire ». Parallèlement à cette constatation, Monsieur C. est confronté à une autre représentation de sa retraite. Il se sent mis hors circuit. Son image personnelle est atteinte. On n'a plus besoin de ses compétences, dit-il. À cette étape de la transition, Monsieur C. révise sa perception de la retraite. Il a vécu le départ de son travail comme une mise hors circuit, un rejet, une mort sociale. Un projet nouveau pointe par contre: se trouver un emploi à l'extérieur, mais l'hésitation l'habite. Il se sent dans un état stationnaire, "renfermé dans une cellule», dira-t-il «jusqu'à ce que je prenne l'erre d'aller ». Il révise ses positions de jadis, celles nommées à la pré-retraite. Il croyait, dit-il, ne pas être touché par la venue de la retraite. Il y a tant à faire, dit-il. D'un point de vue rationnel, il reconnaît qu'il y a des choses à faire mais «avant de prendre la décision et de changer de mode de vie» constate-t-il, "ça dérange ».

La perception subjective du temps change dans l'expérience de Monsieur C. Alors qu'il s'est toujours senti orienté vers l'avenir et les projets, il se surprend depuis quelques mois déjà à régulièrement revenir sur son passé. Il s'empresse par ailleurs à préciser que cette étape de retour est terminée : «y faut lutter contre ça ». Monsieur C. a comme principal objectif de vie de se tenir occupé. Il a des projets dont un voyage dans le «Sud», mais le ton est au conditionnel et sans trop de conviction. Il dit ne pas penser comme tel à la mort «puisque c'est un fait biologique». "Quand on est rendu là, on est rendu là. Faut pas... ça sert à rien de dévier la courbe elle est là. On la prend, c'est tout. » Les pensées de mort font donc partie de son discours. Mais elles sont présentées avec une certaine distance émotionnelle.

Lors du troisième moment de rencontre, dix mois après son départ à la retraite, Monsieur C. s'exprime dans un discours qui rappelle la révolte, l'irascibilité : «La retraite, c'est stupide. »Il évoque d'un ton un peu cynique, l'évolution, dans le temps, de sa façon de percevoir la retraite.

Là, on est de l'autre côté de la clôture. Tout ce que tu sais (expérience de travail), on (employeurs) en a plus besoin. Ça veut pus rien dire [...] Ça compte pus [...] Alors, vous commencez, vous riez. Après ça, on s'habitue à ça. Au bout de six mois, avec le temps, on dit: c'est vrai, ça sert pus, ça pus d'utilité [...] Faut penser que c'est du passé, pis là, on entre dans notre milieu. C'est-à-dire qu'il n'y a pas de milieu.

L'impression de mise hors circuit vécue à la veille du départ à la retraite ne semble pas s'être estompée, au contraire. Tout dans son expérience de retraité est prétexte à critiquer, à dénigrer. Rien n'est attirant. Le seul milieu viable est celui du travail: «si une personne est capable de travailler, elle est mieux occupée». La transition travailretraite semble donc avoir déclenché chez le sujet, à la veille et au lendemain du moment même de l'arrêt, tout un ensemble de remises en question qui, dans le concret de cette troisième rencontre, prennent une coloration fort spéciale. Monsieur C. retourne constamment au passé. Le présent et le futur sont escamotés de sa perspective de vie actuelle: seul le passé occupe la place. Les projets ne sont pas vraiment abordés dans le discours. Son désir et son plaisir dans la vie semblent en hibernation. C'est comme si, par ce retour sur le passé, Monsieur C. cherchait un moyen de mâ̂triser son présent et de surmonter enfin ses difficultés.

\section{UN TRAVAIL D'ADAPTATION PSYCHOSEXUELLE ET SOCIALE}

Au terme de la présentation de ces trois histoires de transition de retraite, il importe de dégager quelques réflexions sur la / les trajectoires des pensées et affects de mort lors du passage travail-retraite. Ces réflexions seront d'abord étayées par les résultats de l'analyse qualitative de l'étude longitudinale servant d'assise au présent article (Thériault, 1986, 1995). Comparée aux trois histoires personnelles ici présentées, la majorité des histoires de retraite relevées dans l'étude longitudinale de base montre qu'au temps pré-retraite la mort est présentée comme une donnée normative chez la majorité des sujets. Le discours est au niveau cognitif. Il est non alarmiste. Il vise explicitement à se rappeler les erreurs de retraite à éviter, dont l'inactivité physique. Au temps intraretraite, la mort est abordée à travers son symbolisme. Elle représente la finalité inévitable de la période de vie en cours. Les per- ceptions du temps sont ici marquées par une contraction du présent. Au temps postretraite, la mort devient personnalisée chez la majorité des sujets: on parle spontanément de sa propre mort. Ce discours n'est pas prolixe, mais il surgit néanmoins spontanément. Les sujets disent repenser malgré eux au travail d'antan, et le futur est mis en question. Ces retours vers le passé suggèrent l'existence d'un processus de « révision de vie » (life review) ; (Levy, 19781979).

Or, une première réflexion veut que les pensées et affects de mort qui surgissent dans le discours des sujets aux temps pré-, intra- ou postretraite soient associés à un processus de réévaluation de vie passée. Ces pensées et affects semblent associés, en retour, à des tentatives de réorientation de la vie présente et future. Pour certains sujets, seules les pensées de mort ont été exprimées, les affects associés à ces pensées étant absents ou non exprimés. Chez les sujets exprimant ces affects associés à la mort, le moment d'apparition pré-, intra- ou postretraite de ces affects a varié selon les sujets. Certains indices nous laissent croire que plus les émotions troubles ou dérangeantes apparaissent tôt dans le processus de transition (p. ex., entrevue pré-retraite), plus les sujets ont de chances de «bénéficier» dès les premiers mois de leur retraite de la réévaluation de vie et réorganisation de vie que ces émotions semblent véhiculer ou entraîner. À la manière d'un vaccin, ces affects de mort dérangeants semblent produire les « anticorps » voulus pour aider les individus à faire face aux changements réels entraînés par le départ et l'adaptation à la retraite. Le travail psychique inhérent à ces affects et/ou au processus de réévaluation de vie aiderait éventuellement les sujets à réinvestir leur présent (avec autre chose que la seule angoisse), à s'ouvrir sur un futur plus prometteur et à se sentir de nouveau des hommes, c'est-à-dire en possession de leur désir.

L'adaptation psychosexuelle de ces préretraités et nouvellement retraités semble être dépendante du moment d'apparition de ces pensées et affects de mort en cours de transition à la retraite. Elle semble également être dépendante du "traitement» que les pré-retraités et retraités ont accordé à ces pensées et affects de mort. Plus particulièrement, il semble que les préretraités et retraités qui ont su retrouver leur désir ou reprendre goût à la vie sont ceux qui ont été ouverts à ces pensées et affects de mort. L'issue du passage travail-retraite semble reliée à la façon dont l'individu accepte de se confronter à l'anxiété de mort.

Les données de la présente étude sur la transition travail-retraite semblent confirmer les données générales formulées par des 
théoriciens existentialistes à l'effet que les pensées et affects de mort ou «l'anxiété de mort» viennent bonifier la vie plutôt que l'appauvrir (exemple Monsieur A.). Il semble qu'une écoute active des individus en transition travail-retraite, offerte par un tiers sensible aux réalités des pré-retraités et retraités, soit souhaitable. Il semble que ce soit au prix d'une prise de conscience, de la verbalisation des pensées de mort et de l'expression des affects qui y sont rattachés que les travailleurs âgés en situation de retraite obligatoire en viennent éventuellement à parfaire leur adaptation psychosexuelle et sociale à la retraite, c'est-à-dire ils reprennent progressivement possession de leur vie - certes revisitée par la perte de la fonction de travailleur institutionnalisé et ils sont en élan vers les autres éléments significatifs de leur existence. Il est reconnu que le travail représente pour l'individu âgé l'un des facteurs externes les plus puissants affectant sa sexualité (Kinsberg, 2000). D'où l'importance de travailler sa perte et les fantasmes de mort qui y sont associés pour favoriser l'adaptation psychosexuelle et sociale du retraité durant la période de retraite.

Les données de cette recherche remet en question les considérations théoriques de Becker (1973) sur le déni de la mort. Suivant l'auteur le déni de la mort, opérationnalisé dans son œuvre sous l'expression projet causa sui ou projet d'éternité, serait toujours actif chez les hommes vieillissants, contrairement à ce qui se passe chez les femmes vieillissantes. La ménopause aurait fourni à ces dernières l'occasion bien tangible, physiologique, de prendre conscience de leur propre finitude humaine. Chez les hommes vieillissants, note Becker (1973), cet indicateur physiologique étant inexistant, leurs illusions d'éternité seraient plus difficiles à abandonner. La mort serait, chez eux, plus facilement déniée. Les données de l'étude longitudinale à la base de cet article donnent toutefois des indications autres : la transition travail-retraite chez les travailleurs âgés en situation de retraite obligatoire semble jouer, au plan psychique, le rôle dévolu à la ménopause chez les femmes. Elle est l'occasion privilégiée d'une saisie dramatique de leur non-immortalité, de leur finitude.

Notons enfin que les réactions des individus rencontrés aux temps pré-, intra- et postretraite rappelleraient en certains points celles observées lors d'un processus de deuil (Thériault, 1994). L'attitude de mise à distance que manifestent la plupart des pré-retraités lors de l'évaluation pré-retraite et l'attitude de révolte rencontrée chez certains sujets de la présente recherche (exemple Monsieur C. au temps post- retraite) rappellent respectivement la première et seconde phases du processus de deuil décrit par Kubler-Ross (1969).

Les résultats d'accentuation des fantasmes de mortalité lors de la période étudiée et les interprétations de deuil attribuées au processus en cours contribuent à considérer la transition étudiée en termes de période à risques. Il semble exister, à la lumière des résultats de cette recherche, un processus d'éveil, d'accentuation et d'aménagement pour le meilleur des fantasmes menaçants lorsque le vécu de retraite se déroule sans embûche particulière. La persistance d'un vécu de menace serait l'indice de la présence d'éléments nuisibles dans le processus. Des degrés très élevés voire nuisibles d'anxiété de mort pourraient ici être à l'œuvre (Rappoport et al., 1993). Une intervention d'aide, propre à alimenter et à appuyer la phase d'introspection accrue qui accompagne le processus de transition, serait particulièrement souhaitable dans ce contexte.

Dans le futur, il serait important de mener cette recherche auprès d'individus inscrits dans différents types de transition travail-retraite (retraite non complète retraite non graduelle, etc.), différentes catégories d'âge (retraite précoce, retraite tardive) et de profession (professionnels, travailleurs indépendants) afin de vérifier si le phénomène d'anxiété de mort se pointe et si les formes qu'il prend aux temps pré-, intra- et postretraite sont comparables à celles relevées chez ces travailleurs manuels en situation de retraite obligatoire. Ce n'est qu'au terme de ces vérifications que les données de cette recherche pourront être généralisées.

\section{Bibliographie}

ATCHLEY, A. (1985). The Social Forces in Later Life, Belmont, CA, Wadsworth.

BECKER, E. (1973). The Denial of Death, New York, Macmillan.

CORMIER, D. (1962). L'échelle d'anxiété IPAT, Montréal, Institut de recherches psychologiques.

DOLLIMORE, J. (1998). Death, Desire and Loss in Western culture, New York, Routledge, 384 p.

ELDER, G.H. (1995). "The life course paradigm: Social change and individual development », dans P. MOEN, G.H. ELDER et K. LUSHER (dir.), Examining Lives in Context; Perspectives on the Ecology of Human Development, Washington, D.C., American Psychological Association, p. 101-139.

GALT, C. et B. HAYSLIP (1998). «Age differences in levels of overt and covert death anxiety », Omega, vol. 37, n 3, p. 187-202.

GUILLEMARD, A.M. (1980). La vieillesse et l'état, Paris, PUF, Politiques, 238 p.
HENDERSON, M. (1990). «Beyond the living will», Gerontologist, vol. 30, $\mathrm{n}^{\circ} 4$ (août), p. 480-485.

KALISH, R.A. (1977). "The role of age in death attitude», Death Education, vol. 1, p. 205-230.

KALISH, R.A. (1987). "The study of death: a psychosocial perspective», dans $H$. WASS, F. BERARDO et R.A. NEIMEYER (dir.), Dying: Facing the Facts, $2^{\mathrm{e}}$ éd., Washington, D.C., Hemisphere.

KINSBERG, S. (2000). "The psychological impact of aging on sexuality and relationships ", Journal of Woman's Health and Gender Based Medecine, vol. 9, p. S33-S38.

KUBLER-ROSS, E. (1969). On Death and Dying, New York, Macmillan.

LEVY, S. (1978-1979). « Temporal experience in the aged: Body integrity and social milieu », International Journal of Aging \& Human Development, vol. 9, $\mathrm{n}^{\circ} 4$, p. 313145.

MOEN, P. (1996). "A life course perspective on retirement, gender, and well-being ", Journal of Occupational Health Psychology, vol. 1, p. 131-144.

NEUGARTEN, B.L., R.J. HAVIGHURST et S. TOBIN (1961). "The measurement of life satisfaction ", Journal of Gerontology, vol. 16, p. 134-143.

RAPPOPORT, H., R.J. FOSSLER, L.S. BROSS et D. GILDEN (1993). "Future time, death, and life purpose among older adults », Death Studies, vol. 17, p. 369-379.

THÉRIAULT, J. (1977). «La retraite, événement de transition", mémoire de maîtrise, option psychologie clinique présentée à l'Université de Montréal, Département de psychologie.

THÉRIAULT, J. (1986). «Le processus de transition à la retraite ", thèse de doctorat, option psychologie clinique, présentée à l'Université de Montréal, Département de psychologie.

THÉRIAULT, J. (1994). «Retirement as a psychosocial transition: Process of adaptation to change », The International Journal of Aging and Human Development, vol. 38, $\mathrm{n}^{\circ} 2$, p. $153-170$.

WASS, H., FM. BERARDO et R.A. NEIMEYER (1987). Dying: Facing the Facts, New York, Hemisphere.

\section{Notes}

1. Les banques de données Eric \& Psyclit ne comptent que neuf articles traitant à la fois des thèmes «death anxiety and retirement » durant la période 1966-2001.

2. Temps de mesure préretraite (six mois avant le départ du travail); temps intra-retraite (quelques semaines après le départ du travail); temps postretraite (sept mois en moyenne après le départ du travail). 